\title{
Stochastic extinction and persistence of a parasite-host epidemiological model
}

\author{
Yuting Liu ${ }^{\mathrm{a}}$, Meijing Shan ${ }^{\mathrm{b}}$, Xinze Lian ${ }^{\mathrm{a}}$, Weiming Wang* a \\ ${ }^{a}$ College of Mathematics and Information Science, Wenzhou University, Wenzhou, 325035 P.R.China \\ ${ }^{b}$ Institute of Information Science and Technology, East China University of Political Science and Law, \\ Shanghai, 201620 P.R.China
}

\begin{abstract}
In this paper, we investigate the stochastic extinction and persistence of a parasite-host epidemiological model. We show that the global dynamics of the stochastic model can be governed by the basic reproduction number $R_{0}^{S}$ : if $R_{0}^{S}<1$, under mild extra conditions, the disease goes to extinction with probability one and the disease-free dynamics occurs; while $R_{0}^{S}>1$, under mild extra conditions, the disease persists and endemic dynamics occurs almost surely, the solutions of the stochastic model fluctuate around the steady state of the deterministic model, and a unique stationary distribution can be found. Based on realistic parameters of Daphnia-microparasite system, numerical simulations have been performed to verify/extend our analytical results. Epidemiologically, we find that: (1) Large environment fluctuations can suppress the outbreak of disease; (2) The distributions are governed by $R_{0}^{S}$; (3) The noise perturbations can be beneficial to control the spread of disease on average. Keywords: Parasite-host model; Extinction; Persistence; Stationary distribution.
\end{abstract}

\section{Introduction}

It is now widely believed that diseases and parasites are responsible for a number of extinctions on islands and on large land masses, and ecologists acknowledge the importance of diseases and parasites in the dynamics of population $[1,2,3,4,5,6]$. Theory on the

Email addresses: liuyuting62@163.com (Yuting Liu), shanmeijing@ecupl.edu.cn (Meijing Shan), xinzelian@163.com (Xinze Lian), weimingwang2003@163.com (Weiming Wang*)

* Author to whom any correspondence should be addressed. 
effects of parasites on host population dynamics has received much attention and focused on issues such as how parasites induce reduction of host fecundity and survival rates change the host population dynamics, and how such dynamics may be applied to predict threats to biodiversity in general and endangered species in particular [2].

In an effort to understand the disease dynamics of microparasite-Daphia magna system, Ebert et al [1] considered a parasite-host epidemiological model of microparasite transmission for a horizontally transmitted parasite that reduces fecundity and survival of its host, which in turn is regulated both by density-dependent birth and by the parasite-induced mortality:

$$
\left\{\begin{array}{l}
\frac{d S}{d t}=r(S+f I)(1-c(S+I))-d S-\beta S I, \\
\frac{d I}{d t}=\beta S I-(\mu+d) I,
\end{array}\right.
$$

where $S$ and $I$ are the densities of the uninfected and the infected hosts (per $100 \mathrm{~mL}$ medium), respectively; $r$ is the maximum per capita birth rate of uninfected hosts; $f$ is the relative fecundity of an infected host; $c$ measures the per capita density-dependent reduction in birth rate $(1 / c$ is also called the carrying capacity if $c \neq 0)$; $d$ is the parasite-independent host background mortality; $\beta$ is the infection rate constant; and $\mu$ is the parasite-induced excess death rate. And Ebert et al. [1] found that strong effects of a parasite on individual host survival and fecundity increased the risk of host population extinction, and the same was true for parasite extinctions. Notice that the disease transmission in model (1) is assumed to be horizontal. Unfortunately, such simple dynamics fails to explain the observed rich outcomes dependent on parameter values and initial population levels. For large populations, individual's finite and often slow movement prevents it to make contact to a large number of individuals in a unit time. Such a mechanism is better described by $\frac{\beta S I}{S+I}$ (called frequency-dependent transmission or standard incidence rate) than $\beta S I$. Conceivably, encounter infection rate makes sense only when the total population is small and steady. In view of this point, Hwang and Kuang [2] formulated the following model to improve mod- 
el (1):

$$
\left\{\begin{array}{l}
\frac{d S}{d t}=r(S+f I)(1-c(S+I))-d S-\frac{\beta S I}{N} \\
\frac{d I}{d t}=\frac{\beta S I}{N}-(\mu+d) I
\end{array}\right.
$$

where $N=S+I$ is the total population size. And the most important finding of Hwang and Kuang [2] is that the origin can be an attractor (global) for model (2), which may explain the often-observed deterministic extinctions of hosts. This host extinction dynamics has resulted from the degeneracy of the origin due to the ratio-dependent infection term.

Furthermore, considering the emigration of the susceptible, based on the results of $[1,2]$, Berezovskaya et al. [4] explored an epidemic model incorporating demographic and epidemiological processes:

$$
\left\{\begin{array}{l}
\frac{d S}{d t}=r N\left(1-\frac{N}{K}\right)-\frac{\beta S I}{N}-(\mu+m) S, \\
\frac{d I}{d t}=\frac{\beta S I}{N}-(\mu+d) I,
\end{array}\right.
$$

where $K=1 / c$ is the carrying capacity, $m$ denotes the per-capita emigration rate of the infectious.

Define two threshold parameters, one is the basic reproductive number

$$
R_{0}=\frac{\beta}{\mu+d},
$$

and the other is the basic demographic reproduction number

$$
R_{d}=\frac{r}{\mu+m}
$$

Basically, $R_{d}>1$ implies that each founder individual leaves more than one descendant on average before it dies, that is, a critical mass of individuals for the disease to spread may be supported. While if $R_{d}<1$ implies that births will exceed deaths at the beginning of the process and the population will not survive, that is, no critical mass of individuals may be supported for the disease to spread [4]. Hence, in the remainder of this paper, we assume that $R_{d}>1$.

Based on the results in [4], qualitative properties for model (3) are summarized in the following two statements. 
Theorem 1.1. If $R_{0}<1$, then the disease-free steady state

$$
E_{0}=\left(S_{*}, 0\right)=\left(K\left(R_{d}-1\right) / R_{d}, 0\right)
$$

is globally asymptotically stable. While if $R_{0}>1, E_{0}$ is unstable.

Theorem 1.2. If $R_{0}>1$ and $R_{d}>\frac{R_{0}(d+m)+m-d}{R_{0}(\mu+m)}$, there exists a unique endemic state $E^{*}=\left(S^{*}, I^{*}\right)$ which is globally asymptotically stable, where

$$
\begin{aligned}
S^{*} & =\frac{K\left(m R_{0} R_{d}+\mu R_{0} R_{d}-d R_{0}-\mu R_{0}+d-m\right)}{R_{d} R_{0}^{2}(\mu+m)}, \\
I^{*} & =\frac{K\left(m R_{0} R_{d}+\mu R_{0} R_{d}-d R_{0}-\mu R_{0}+d-m\right)\left(R_{0}-1\right)}{R_{d} R_{0}^{2}(\mu+m)} .
\end{aligned}
$$

On the other hand, stochastic models could be a more appropriate way of modeling epidemics in many circumstances $[7,8,9,10,11,12,13,14,15,16,17,18,19,20,21,22$, $23,24,25]$. To incorporate the effect of environmental fluctuations, here we formulate a stochastic differential equation (SDE) model by introducing the multiplicative noise terms into the growth equations of both the susceptible and the infectious populations [7] and assume that the disease transmission coefficient $\beta$ will fluctuate around some average value due to continuous fluctuation in the environment. And we introduce randomness into the deterministic model (3) by perturbing $\beta$ by $\beta+\sigma \zeta(t)$ to model (3):

$$
\left\{\begin{array}{l}
\frac{d S}{d t}=r N\left(1-\frac{N}{K}\right)-(\beta+\sigma \zeta(t)) \frac{S I}{N}-(\mu+m) S, \\
\frac{d I}{d t}=(\beta+\sigma \zeta(t)) \frac{S I}{N}-(\mu+d) I,
\end{array}\right.
$$

where $\zeta(t)$ is a Gaussian white noise and characterized by:

$$
\langle\zeta(t)\rangle=0, \quad\left\langle\zeta(t) \zeta\left(t^{\prime}\right)\right\rangle=\delta\left(t-t^{\prime}\right),
$$

here $\langle\cdot\rangle$ denotes ensemble average and $\delta(\cdot)$ is the Dirac- $\delta$ function. $\sigma_{1}$ and $\sigma_{2}$ denote the intensity of environmental forcing. And we can rewrite model (7) into the form of stochastic differential equations as follows:

$$
\left\{\begin{array}{l}
d S=\left(r N\left(1-\frac{N}{K}\right)-\frac{\beta S I}{N}-(\mu+m) S\right) d t-\frac{\sigma S I}{N} d B(t), \\
d I=\left(\frac{\beta S I}{N}-(\mu+d) I\right) d t+\frac{\sigma S I}{N} d B(t),
\end{array}\right.
$$


where $B(t)$ is the standard one-dimensional independent Wiener process defined over the complete probability space $\left(\Omega, \mathcal{F},\left\{\mathcal{F}_{t}\right\}_{t \geq 0}, \mathbb{P}\right)$, and the relations between the white noise terms and Wiener process are defined by $d B(t)=\zeta(t) d t$.

Define a bounded set $\Delta$ as follows:

$$
\Delta=\left\{(S, I) \in \mathbb{R}_{+}^{2}: 0<S+I<K\right\}
$$

one can easyly obtain the following result about the existence of the positive global solution of the SDE model (8):

Theorem 1.3. Let $\left(S_{0}, I_{0}\right) \in \Delta$, then the system admits a unique positive solution $(S(t), I(t))$ on $t \geq 0$ and will remains in $\Delta$ with probability one.

The proof of this Theorem 1.3 is rather standard and hence is omitted.

The main goal of this paper is to investigate how environment fluctuations affect disease dynamics through studying the global dynamics of the SDE model. The rest of this article is organized as follows: In Section 2, we carry out the stochastic disease-free dynamics. In section 3, we investigate the stochastic endemic dynamics and the existence of a unique stationary distribution. In section 4 , we give some numerical results and make a comparative analysis of the dynamics of the model with deterministic and stochastic environments. In the last section, Section 5, we provide a brief discussion and the summary of the main results.

\section{Stochastic extinction}

One of the main concerns in epidemiology is how we can regulate the disease dynamics so that the disease will be eradicated in a long term. First of all, we give the following lemma:

Lemma 2.1. [26] Let $f \in C[[0, \infty) \times \Omega,(0, \infty)]$.

(i) If there exist positive constants $\lambda_{0}, \lambda$ such that

$$
\log f(t) \geq \lambda t-\lambda_{0} \int_{0}^{t} f(s) d s+F(t), \quad \text { a.s. }
$$


for all $t \geq 0$, where $F \in C[[0, \infty) \times \Omega, \mathbb{R}]$ and $\lim _{t \rightarrow \infty} \frac{F(t)}{t}=0$ a.s., then

$$
\lim _{t \rightarrow \infty} \inf \frac{1}{t} \int_{0}^{t} f(s) d s \geq \frac{\lambda}{\lambda_{0}} \text { a.s. }
$$

(ii) If there exist positive constants $\lambda_{0}$ and $\lambda$ such that

$$
\log f(t) \leq \lambda t-\lambda_{0} \int_{0}^{t} f(s) d s+F(t), \quad \text { a.s. }
$$

for all $t \geq 0$, where $F \in C[[0, \infty) \times \Omega, \mathbb{R}]$ and $\lim _{t \rightarrow \infty} \frac{F(t)}{t}=0$ a.s., then

$$
\lim _{t \rightarrow \infty} \sup \frac{1}{t} \int_{0}^{t} f(s) d s \leq \frac{\lambda}{\lambda_{0}} \text { a.s. }
$$

The proof of the lemma above can be found in [26] and hence is omitted here.

Next, we define a threshold parameter named the basic reproduction number for the SDE model (8):

$$
R_{0}^{S}:=R_{0}-\frac{\sigma^{2}}{2(\mu+d)}
$$

Then, we can obtain the following theorem about the extinction of the disease for the SDE model (8).

Theorem 2.2. Let $(S(t), I(t))$ be the solution of model (8) with initial value $(S(0), I(0)) \in \Delta$. If

(i) $\sigma^{2}>\max \left\{\frac{\beta^{2}}{2(\mu+d)}, \beta\right\}$

or

(ii) $R_{0}^{S}<1$ and $\sigma^{2} \leq \beta$

hold, then

$$
\begin{aligned}
& \lim _{t \rightarrow \infty} \sup \frac{\log I(t)}{t} \leq(\mu+d)\left(R_{0}^{S}-1\right)<0 \quad \text { a.s., } \\
& \lim _{t \rightarrow \infty} \sup \frac{1}{t} \int_{0}^{t} S(s) d s \leq K\left(R_{d}-1\right) / R_{d} \quad \text { a.s. }
\end{aligned}
$$

namely, the infectious $I(t)$ tends to zero exponentially almost surely. That is, the disease will die out with probability one. 
Proof. Applying the Ito's Formula to system (8) leads to

$$
\begin{aligned}
d(\log I(t)) & =\left[\frac{\beta S}{S+I}-(\mu+d)-\frac{1}{2} \cdot\left(\frac{\sigma S}{S+I}\right)^{2}\right] d t+\frac{\sigma S}{S+I} d B(t) \\
& =\left[-\frac{\sigma^{2}}{2}\left(\frac{S}{S+I}-\frac{\beta}{\sigma^{2}}\right)^{2}+\frac{\beta^{2}}{2 \sigma^{2}}-(\mu+d)\right] d t+\frac{\sigma S}{S+I} d B(t) \\
& =f(I) d t+\frac{\sigma S}{S+I} d B(t),
\end{aligned}
$$

here $f:(0,1) \rightarrow \mathbb{R}$ is defined by

$$
f(x)=-\frac{\sigma^{2}}{2}\left(x-\frac{\beta}{\sigma^{2}}\right)^{2}+\frac{\beta^{2}}{2 \sigma^{2}}-(\mu+d) \quad \text { and } \quad x=\frac{S}{S+I} .
$$

Integrating (15) from 0 to $t$ and dividing by $t$ on both sides, we can get

$$
\frac{\log I(t)}{t} \leq \frac{\log I(0)}{t}+\frac{1}{t} \int_{0}^{t} f(x) d s+\frac{1}{t} \int_{0}^{t} \frac{\sigma S}{S+I} d B(s) .
$$

(i) Compute

$$
f(x)=-\frac{\sigma^{2}}{2}\left(x-\frac{\beta}{\sigma^{2}}\right)^{2}+\frac{\beta^{2}}{2 \sigma^{2}}-(\mu+d) \leq \frac{\beta^{2}}{2 \sigma^{2}}-(\mu+d),
$$

which is negative by the condition $\sigma^{2}>\max \left\{\frac{\beta^{2}}{2(\mu+d)}, \beta\right\}$. It therefore follows from and (18) that

$$
\frac{\log I(t)}{t} \leq-(\mu+d)+\frac{\beta^{2}}{2 \sigma^{2}}+\frac{M_{1}}{t}+\frac{\log I(0)}{t}
$$

where $M_{1}:=\sigma \int_{0}^{t} \frac{S}{S+I} d B(s)$. By the large number theorem for martingales, we have

$$
\lim _{t \rightarrow \infty} \frac{M_{1}}{t}=0 \quad \text { a.s.. }
$$

Taking the limit superior of both sides, we can obtain the desired assertion (19) as

$$
\lim _{t \rightarrow \infty} \sup \frac{\log I(t)}{t} \leq-(\mu+d)+\frac{\beta^{2}}{2 \sigma^{2}}<0 \quad \text { a.s.. }
$$

(ii) Now we focus on the case of $\sigma^{2} \leq \beta$. From (18) and the condition $\sigma^{2} \leq \beta$, we can know that

$$
\bar{x}=\frac{\beta}{\sigma^{2}} \geq 1,
$$


and then $f(x)$ takes its maximum value

$$
f(\hat{x})=f(1)=\beta-(\mu+d)-\frac{\sigma^{2}}{2}=\left(R_{0}^{S}-1\right)(\mu+d) .
$$

It follows from (17) and (22) that

$$
\frac{\log I(t)}{t} \leq\left(R_{0}^{S}-1\right)(\mu+d)+\frac{M_{1}}{t}+\frac{\log I(0)}{t} .
$$

If $R_{0}^{S}<1$, then

$$
\limsup _{t \rightarrow \infty} \frac{\log I(t)}{t} \leq\left(R_{0}^{S}-1\right)(\mu+d)<0 \text { a.s.. }
$$

From (21) and (23), we can get that there is some constant $\phi>0$ such that

$$
\limsup _{t \rightarrow \infty} \frac{\log I(t)}{t} \leq-\phi
$$

That is, for any arbitrary small constant $0<\varepsilon<\min \left(\frac{1}{2}, \frac{\phi}{2}\right)$, there exists a positive constant $T_{1}=T_{1}(w)$ and a set $\Omega_{\varepsilon}$ such that $P\left(\Omega_{\varepsilon}\right) \geq 1-\varepsilon$ and $\log I(t) \leq \frac{-\phi t}{2}$ for $t \geq T_{1}, w \in \Omega_{\varepsilon}$, and so $I(t) \leq e^{\frac{-\phi t}{2}}$. Therefore

$$
\limsup _{t \rightarrow \infty} I(t) \leq 0 \text { a.s. }
$$

which together with the positivity of the solution (c.f., Theorem 1.3) and the results in (21) and (23) imply that

$$
\lim _{t \rightarrow \infty} I(t)=0 \text { a.s. }
$$

which means that $I(t)$ goes to extinct exponentially a.s..

On the other hand, note that

$$
d S=\left(r N\left(1-\frac{N}{K}\right)-\frac{\beta S I}{N}-(\mu+m) S\right) d t-\frac{\sigma S I}{N} d B(t),
$$

and nondimensionalize with the following scaling:

$$
\tilde{S}=S-S_{*}=S-K\left(R_{d}-1\right) / R_{d}
$$

where $S_{*}$ is defined as in (6), hence (25) can be rewritten as:

$$
d \tilde{S}=-\frac{r}{K} S_{*} \tilde{S}-\frac{r \tilde{S}^{2}}{K}+\delta(S, I)-\frac{\sigma S I}{N} d B(t) \leq-\frac{r}{K} S_{*} \tilde{S}+\delta(S, I)-\frac{\sigma S I}{N} d B(t),
$$


where $\delta(S, I)=r\left(1-\frac{S+I}{K}-\frac{\beta S}{S+I}-\frac{S}{K}\right) I \leq r I$, and

$$
\frac{r}{K} S_{*} \frac{1}{t} \int_{0}^{t} \tilde{S}(s) d s \leq \frac{1}{t} \int_{0}^{t} \delta(S(s), I(s)) d s-\frac{1}{t} \int_{0}^{t} \frac{\sigma S(s) I(s)}{N(s)} d s-\frac{\tilde{S}(t)-\tilde{S}(0)}{t},
$$

then according to (24) and the large number theorem for martingales, we can get

$$
\limsup _{t \rightarrow \infty} \frac{1}{t} \int_{0}^{t} \delta(S(s), I(s)) d s=0 \quad \text { a.s. }
$$

and

$$
\limsup _{t \rightarrow \infty} \frac{1}{t} \int_{0}^{t} \frac{\sigma S(s) I(s)}{N(s)} d s=0 \quad \text { a.s.. }
$$

Hence

$$
\lim \sup _{t \rightarrow \infty} \frac{1}{t} \int_{0}^{t} \tilde{S}(s) d s \leq 0
$$

which implies that

$$
\lim _{t \rightarrow \infty} \sup \frac{1}{t} \int_{0}^{t} S(s) d s \leq K\left(R_{d}-1\right) / R_{d} \text { a.s. }
$$

This ends the proof.

\section{Stochastic persistence}

The deterministic SIS model (3) is globally at its endemic equilibrium $E^{*}=\left(S^{*}, I^{*}\right)$ whenever $R_{0}=\frac{\beta}{\mu+d}>1$. Since model (8) is the perturbed system of model (3), it seems reasonable to consider the disease will prevail if the solution of model (8) has the ergodic property. In the following, we first consider the persistence of the SDE model (8).

\subsection{Persistence of the disease}

Theorem 3.1. If $\sigma^{2}<\beta, r>\mu+\delta_{2}$ and $1<R_{0}^{S}<1+\frac{\left(r-\mu-\delta_{2}\right)^{2}\left(\beta-\sigma^{2}\right)}{r\left(r-\mu-\delta_{1}\right)^{2}(\mu+d)}$, here $\delta_{1}=\min \{m, d\}, \delta_{2}=\max \{m, d\}$, then for any given initial values $(S(0), I(0)) \in \Delta$, the solution of model (8) obeys

$$
\begin{gathered}
\lim _{t \rightarrow \infty} \inf \frac{1}{t} \int_{0}^{t} I(s) d s \geq \frac{K(\mu+d)\left(R_{0}^{S}-1\right)\left(r-\mu-\delta_{2}\right)}{\beta r} \text { a.s. } \\
\lim _{t \rightarrow \infty} \sup \frac{1}{t} \int_{0}^{t} I(s) d s \leq \frac{K(\mu+d)\left(R_{0}^{S}-1\right)}{\beta-\sigma^{2}} \text { a.s. }
\end{gathered}
$$


and

$$
\begin{aligned}
& \lim _{t \rightarrow \infty} \inf \frac{1}{t} \int_{0}^{t} S(s) d s \geq \frac{K\left(r-\mu-\delta_{2}\right)^{2}}{r(r-\mu-m)}-\frac{K(\mu+d)\left(R_{0}^{S}-1\right)(r-\mu-d)}{\left(\beta-\sigma^{2}\right)(r-\mu-m)} \text { a.s. } \\
& \lim _{t \rightarrow \infty} \sup \frac{1}{t} \int_{0}^{t} S(s) d s \leq \frac{K r}{r-\mu-m}-\frac{K(\mu+d)\left(R_{0}^{S}-1\right)\left(r-\mu-\delta_{2}\right)(r-\mu-d)}{\beta r(r-\mu-m)} \text { a.s. }
\end{aligned}
$$

Proof. If $\delta_{2}=\max (m, d)$, the total population in model (8) verifies the equation:

$$
d N\left(r N\left(1-\frac{N}{K}\right)-(\mu+m) S-(\mu+d) I\right) d t \geq N\left(r-\mu-\delta_{2}-\frac{r}{K} N\right) d t
$$

Letting $t \rightarrow+\infty$, we can get

$$
\liminf _{t \rightarrow \infty} N \geq \frac{K\left(r-\mu-\delta_{2}\right)}{r} .
$$

By using Ito's formula for the second equation of model (8), we have

$$
d(\log I(t))=\left(\frac{\beta S}{S+I}-(\mu+d)-\frac{1}{2}\left(\frac{\sigma S}{S+I}\right)^{2}\right) d t+\frac{\sigma S}{S+I} d B(t) .
$$

Integrating this from 0 to $t$ and dividing by $t$ on both sides, we have

$$
\begin{aligned}
\frac{\log I(t)}{t} & =\beta-\frac{\beta}{t} \int_{0}^{t} \frac{I(s)}{S(s)+I(s)} d s-(\mu+d)-\frac{\sigma^{2}}{2} \frac{1}{t} \int_{0}^{t}\left(\frac{S(s)}{S(s)+I(s)}\right)^{2} d s+\frac{\log I(0)}{t}+\frac{M_{1}}{t} \\
& \geq-\beta \frac{1}{t} \int_{0}^{t} \frac{I(s)}{S(s)+I(s)} d s+\beta-(\mu+d)-\frac{\sigma^{2}}{2}+\frac{\log I(0)}{t}+\frac{M_{1}}{t} \\
& \geq-\frac{\beta r}{K\left(r-\mu-\delta_{2}\right)} \frac{1}{t} \int_{0}^{t} I(s) d s+\beta-(\mu+d)-\frac{\sigma^{2}}{2}+\frac{\log I(0)}{t}+\frac{M_{1}}{t} \\
& =-\frac{\beta r}{K\left(r-\mu-\delta_{2}\right)} \frac{1}{t} \int_{0}^{t} I(s) d s+(\mu+d)\left(R_{0}^{S}-1\right)+\frac{\log I(0)}{t}+\frac{M_{1}}{t}
\end{aligned}
$$

where $M_{1}=\sigma \int_{0}^{t} \frac{S(s)}{S(s)+I(s)} d B(s)$ and $\lim _{t \rightarrow \infty} \frac{M_{1}}{t}=0$ a.s.. Then together with Lemma 2.1(i), we can get

$$
\liminf _{t \rightarrow \infty} \frac{1}{t} \int_{0}^{t} I(s) d s \geq \frac{K(\mu+d)\left(R_{0}^{S}-1\right)\left(r-\mu-\delta_{2}\right)}{\beta r} \text { a.s.. }
$$


On the other hand,

$$
\begin{aligned}
\frac{\log I(t)}{t}= & \beta-\frac{\beta}{t} \int_{0}^{t} \frac{I(s)}{S(s)+I(s)} d s-\frac{\sigma^{2}}{2} \frac{1}{t} \int_{0}^{t}\left(\frac{S(s)}{S(s)+I(s)}\right)^{2} d s+\frac{\log I(0)}{t}+\frac{M_{1}}{t}-(\mu+d) \\
= & -\frac{\beta}{t} \int_{0}^{t} \frac{I(s)}{S(s)+I(s)} d s+\beta-\frac{\sigma^{2}}{2} \frac{1}{t} \int_{0}^{t}\left(1-\frac{2 I(s)}{S(s)+I(s)}+\left(\frac{I(s)}{S(s)+I(s)}\right)^{2}\right) d t \\
& +\frac{\log I(0)}{t}+\frac{M_{1}}{t}-(\mu+d) \\
\leq & \beta-\frac{\sigma^{2}}{2}-(\mu+d)-\left(\beta-\sigma^{2}\right) \frac{1}{t} \int_{0}^{t} \frac{I(s)}{S(s)+I(s)} d s+\frac{\log I(0)}{t}+\frac{M_{1}}{t} \\
\leq & \beta-\frac{\sigma^{2}}{2}-(\mu+d)-\frac{\left(\beta-\sigma^{2}\right)}{K} \frac{1}{t} \int_{0}^{t} I(s) d s+\frac{\log I(0)}{t}+\frac{M_{1}}{t} \\
= & (\mu+d)\left(R_{0}^{S}-1\right)-\frac{\left(\beta-\sigma^{2}\right)}{K} \frac{1}{t} \int_{0}^{t} I(s) d s+\frac{\log I(0)}{t}+\frac{M_{1}}{t} .
\end{aligned}
$$

Then according to Lemma 2.1(ii), we can get

$$
\limsup _{t \rightarrow \infty} \frac{1}{t} \int_{0}^{t} I(s) d s \leq \frac{K(\mu+d)\left(R_{0}^{S}-1\right)}{\beta-\sigma^{2}} \quad \text { a.s.. }
$$

Considering (3) again, we can get

$$
\frac{d N}{d t}=r N\left(1-\frac{N}{K}\right)-(\mu+m) S-(\mu+d) I \text { a.s.. }
$$

It then follows that

$$
\begin{aligned}
d N & =\left((r-\mu-m) S+(r-\mu-d) I-\frac{r N^{2}}{K}\right) d t \\
& \leq\left((r-\mu-m) S+(r-\mu-d) I-\frac{K\left(r-\mu-\delta_{2}\right)^{2}}{r}\right) d t
\end{aligned}
$$

then it is easy to obtain

$$
\frac{1}{t} \int_{0}^{t} S(s) d s \geq \frac{K\left(r-\mu-\delta_{2}\right)^{2}}{r(r-\mu-m)}-\frac{(r-\mu-d)}{r-\mu-m} \frac{1}{t} \int_{0}^{t} I(s) d s+\frac{(S(t)+I(t))-(S(0)+I(0))}{t(r-\mu-m)} .
$$

From (30), we can deduce that for any $\eta_{1}>0\left(\eta_{1}<\frac{K(\mu+d)\left(R_{0}^{S}-1\right)}{\beta-\sigma^{2}}\right)$, there is a $T_{2}(w)>0$ such that

$$
\frac{1}{t} \int_{0}^{t} I(s) d s \leq \frac{K(\mu+d)\left(R_{0}^{S}-1\right)}{\beta-\sigma^{2}}+\eta_{1}
$$


for $t \geq T_{2}(w)$. Then

$$
\begin{gathered}
\frac{1}{t} \int_{0}^{t} S(s) d s \geq \frac{K\left(r-\mu-\delta_{2}\right)^{2}}{r(r-\mu-m)}-\frac{r-\mu-d}{r-\mu-m}\left(\frac{K(\mu+d)\left(R_{0}^{S}-1\right)}{\beta-\sigma^{2}}+\eta_{1}\right) \\
+\frac{(S(t)+I(t))-(S(0)+I(0))}{t(r-\mu-m)}
\end{gathered}
$$

for $t \geq T_{2}(w)$. Letting $t \rightarrow \infty$, with $\eta_{1}$ arbitrary, we can get

$$
\lim _{t \rightarrow \infty} \inf \frac{1}{t} \int_{0}^{t} S(s) d s \geq \frac{K\left(r-\mu-\delta_{2}\right)^{2}}{r(r-\mu-m)}-\frac{K(\mu+d)\left(R_{0}^{S}-1\right)(r-\mu-d)}{\left(\beta-\sigma^{2}\right)(r-\mu-m)} \text { a.s. }
$$

In addition, from (3), one can obtain:

$$
\frac{d N}{d t}=(r-\mu-m) S+(r-\mu-d) I-\frac{r N^{2}}{K} \geq(r-\mu-m) S+(r-\mu-d) I-K r .
$$

Then we can get the following inequality

$$
\frac{1}{t} \int_{0}^{t} S(s) d s \leq \frac{K r}{(r-\mu-m)}-\frac{(r-\mu-d)}{r-\mu-m} \frac{1}{t} \int_{0}^{t} I(s) d s+\frac{(S(t)+I(t))-(S(0)+I(0))}{t}
$$

From (29), we can get $\forall \eta_{2}>0\left(\eta_{2}<\frac{K(\mu+d)\left(R_{0}^{S}-1\right)\left(r-\mu-\delta_{2}\right)}{\beta r}\right.$, there is a $T\left(w_{2}\right)$ such that

$$
\frac{1}{t} \int_{0}^{t} I(s) d s \geq \frac{K(\mu+d)\left(R_{0}^{S}-1\right)\left(r-\mu-\delta_{2}\right)}{\beta r}-\eta_{2}
$$

then by similar arguments, we have

$$
\lim _{t \rightarrow \infty} \sup \frac{1}{t} \int_{0}^{t} S(s) d s \leq \frac{K r}{r-\mu-m}-\frac{K(\mu+d)\left(R_{0}^{S}-1\right)\left(r-\mu-\delta_{2}\right)(r-\mu-d)}{\beta r(r-\mu-m)} \text { a.s. }
$$

This completes the proof of Theorem 3.1.

\subsection{Stochastic asymptotic stability}

Theorem 3.2. If the following two conditions hold,

(i) $R_{0}^{S}>1, R_{d}>\frac{R_{0}(d+m)+m-d}{R_{0}(\mu+m)}$;

(ii) $r>\max \left\{\mu+2 \delta_{2}-\delta_{1}, \mu+2 \delta_{1}-\delta_{2}+\frac{\sigma^{2}}{2}\right\}$; 
where $\delta_{1}=\min \{m, d\}, \delta_{2}=\max \{m, d\}$. Then for any initial value $(S(0), I(0)) \in \Delta$, the solution $(S(t), I(t))$ of the SDE model (8) has the property:

$$
\begin{aligned}
\lim _{t \rightarrow \infty} \sup & \frac{1}{t} \int_{0}^{t}\left(\left(S-\frac{2\left(r-\mu-2 \delta_{2}+m\right)}{2\left(r-\mu-2 \delta_{2}+m\right)-\sigma^{2}} S^{*}\right)^{2}+\left(I-\frac{2\left(r-\mu-2 \delta_{2}+d\right)}{2\left(r-\mu-2 \delta_{2}+d\right)-\sigma^{2}} I^{*}\right)^{2}\right) d \tau \\
& \leq \frac{\Psi}{\Theta}
\end{aligned}
$$

where

$$
\begin{aligned}
\Psi= & \frac{\left(r-\mu-2 \delta_{2}+m\right)}{2\left(r-\mu-2 \delta_{2}+m\right)-\sigma^{2}} S^{* 2}+\frac{\left(r-\mu-2 \delta_{2}+d\right)}{2\left(r-\mu-2 \delta_{2}+d\right)-\sigma^{2}} I^{* 2} \\
& +\frac{\left(m+d+2 r-2 \mu-4 \delta_{2}\right)\left(S^{*}+I^{*}\right)}{2 \beta} \sigma^{2} I^{*}, \\
\Theta= & r-\mu-2 \delta_{2}+\delta_{1}+\frac{\sigma^{2}}{2},
\end{aligned}
$$

Proof. Since $R_{0}^{S}>1$, hence $R_{0}=R_{0}^{S}+\frac{\sigma^{2}}{2(\mu+d)}>1$, there is an endemic equilibrium $E^{*}=\left(S^{*}, I^{*}\right)$ of model $(3)$, then

$$
\left\{\begin{array}{l}
r N^{*}\left(1-\frac{N^{*}}{K}\right)=\beta \frac{S^{*} I^{*}}{N^{*}}+(\mu+m) S^{*}, \\
\beta \frac{S^{*} I^{*}}{N^{*}}=(\mu+d) I^{*}
\end{array}\right.
$$

Hence

$$
r N^{*}\left(1-\frac{N^{*}}{K}\right)=(\mu+m) S^{*}+(\mu+d) I^{*}
$$

Set

$$
V(S, I)=\frac{1}{2}\left(S-S^{*}+I-I^{*}\right)^{2}+\lambda\left(I-I^{*}-I^{*} \log \frac{I}{I^{*}}\right):=V_{1}(S, I)+\lambda V_{2}(I),
$$

where $\lambda$ is positive constants to be determined later, and $V$ is a nonnegative $C^{2}$ function. 
Considering (38), (39) and (34) together, by using the Ito's formula, we can obtain

$$
\begin{aligned}
d V_{1}= & {\left[\left(S-S^{*}+I-I^{*}\right)\left(r N\left(1-\frac{N}{K}\right)-(\mu+m) S-(\mu+d) I\right)+\frac{\sigma^{2} S^{2} I^{2}}{N^{2}}\right] d t } \\
= & {\left[( S - S ^ { * } + I - I ^ { * } ) \left(r N\left(1-\frac{N}{K}\right)-(\mu+m)\left(S-S^{*}\right)-r N^{*}\left(1-\frac{N^{*}}{K}\right)\right.\right.} \\
& \left.-(\mu+d)\left(I-I^{*}\right)+\frac{\sigma^{2} S^{2} I^{2}}{N^{2}}\right] d t \\
= & {\left[r\left(S-S^{*}+I-I^{*}\right)^{2}-\frac{r}{K}\left(S-S^{*}+I-I^{*}\right)^{2}\left(S+I+S^{*}+I^{*}\right)-(\mu+m)\left(S-S^{*}\right)^{2}\right.} \\
& \left.-(\mu+d)\left(I-I^{*}\right)^{2}-(2 \mu+m+d)\left(S-S^{*}\right)\left(I-I^{*}\right)+\frac{\sigma^{2} S^{2} I^{2}}{(S+I)^{2}}\right] d t \\
\leq & {\left[-\left(r-\mu-2 \delta_{2}+m\right)\left(S-S^{*}\right)^{2}-\left(r-\mu-2 \delta_{2}+d\right)\left(I-I^{*}\right)^{2}\right.} \\
& \left.-\left(m+d+2 r+2 \mu-4 \delta_{2}\right)\left(S-S^{*}\right)\left(I-I^{*}\right)+\frac{\sigma^{2} S^{2} I^{2}}{(S+I)^{2}}\right] d t,
\end{aligned}
$$

and

$$
\begin{aligned}
d V_{2}= & \left(\left(I-I^{*}\right)\left(\frac{\beta S}{N}-(\mu+d)+\frac{\sigma^{2} S^{2} I^{*}}{2 N^{2}}\right) d t+\frac{\sigma S\left(I-I^{*}\right)}{N} d B(t)\right. \\
= & \left(\left(I-I^{*}\right)\left(\frac{\beta S}{S+I}-\frac{\beta S^{*}}{S^{*}+I^{*}}+\frac{\sigma^{2} S^{2} I^{*}}{2(S+I)^{2}}\right) d t+\frac{\sigma S\left(I-I^{*}\right)}{(S+I)} d B(t)\right. \\
= & \left(\left(I-I^{*}\right)\left(\frac{\beta\left(S-S^{*}\right)}{S^{*}+I^{*}}+\frac{\beta S\left(\left(S^{*}-S\right)+\left(I^{*}-I\right)\right)}{\left(S^{*}+I^{*}\right)(S+I)}+\frac{\sigma^{2} S^{2} I^{*}}{2(S+I)^{2}}\right) d t+\frac{\sigma S\left(I-I^{*}\right)}{S+I} d B(t)\right. \\
\leq & \left(-\frac{\beta S}{\left(S^{*}+I^{*}\right)(S+I)}\left(S-S^{*}\right)\left(I-I^{*}\right)+\frac{\beta}{S^{*}+I^{*}}\left(S-S^{*}\right)\left(I-I^{*}\right)+\frac{\sigma^{2} S^{2} I^{*}}{2(S+I)^{2}}\right) d t \\
& +\frac{\sigma S\left(I-I^{*}\right)}{S+I} d B(t) \\
\leq & \left(\frac{\beta}{S^{*}+I^{*}}\left(S-S^{*}\right)\left(I-I^{*}\right) \operatorname{sign}\left(S-S^{*}\right)\left(I-I^{*}\right)+\frac{\sigma^{2} S^{2} I^{*}}{2(S+I)^{2}}\right) d t+\frac{\sigma S\left(I-I^{*}\right)}{(S+I)} d B(t) .
\end{aligned}
$$

Adopt

$$
\lambda=\frac{\left(m+d+2 r-2 \mu-4 \delta_{2}\right)\left(S^{*}+I^{*}\right)}{\beta}>0,
$$


then

$$
\begin{aligned}
d V \leq & {\left[-\left(r-\mu-2 \delta_{2}+m\right)\left(S-S^{*}\right)^{2}-\left(r-\mu-2 \delta_{2}+d\right)\left(I-I^{*}\right)^{2}\right.} \\
& \left.+\frac{\sigma^{2} S^{2} I^{2}}{(S+I)^{2}}+\frac{\lambda \sigma^{2} S^{2}}{2(S+I)^{2}} I^{*}\right] d t+\frac{\lambda \sigma S}{S+I}\left(I-I^{*}\right) d B(t) \\
= & L V d t+\frac{\lambda \sigma S}{(S+I)}\left(I-I^{*}\right) d B(t),
\end{aligned}
$$

Using the facts that $S(t)>0$ and $I(t)>0$, we can get

$$
\frac{S^{2} I^{2}}{(S+I)^{2}} \leq \frac{S^{2} I^{2}}{S^{2}+I^{2}} \leq \frac{S^{2}+I^{2}}{2}, \quad \frac{S^{2}}{(S+I)^{2}}<1
$$

hence

$$
\begin{aligned}
L V= & -\left(r-\mu-2 \delta_{2}+m\right)\left(S-S^{*}\right)^{2}-\left(r-\mu-2 \delta_{2}+d\right)\left(I-I^{*}\right)^{2}+\frac{\sigma^{2} S^{2} I^{2}}{(S+I)^{2}}+\frac{\lambda \sigma^{2} S^{2}}{2(S+I)^{2}} I^{*} \\
\leq & -\left(r-\mu-2 \delta_{2}+m\right)\left(S-S^{*}\right)^{2}-\left(r-\mu-2 \delta_{2}+d\right)\left(I-I^{*}\right)^{2}+\frac{\sigma^{2}\left(S^{2}+I^{2}\right)}{2}+\frac{\lambda \sigma^{2}}{2} I^{*} \\
= & -\left(r-\mu-2 \delta_{2}+m-\frac{\sigma^{2}}{2}\right)\left(S-\frac{2\left(r-\mu-2 \delta_{2}+m\right)}{2\left(r-\mu-2 \delta_{2}+m\right)-\sigma^{2}} S^{*}\right)^{2} \\
& -\left(r-\mu-2 \delta_{2}+d-\frac{\sigma^{2}}{2}\right)\left(I-\frac{2\left(r-\mu-2 \delta_{2}+d\right)}{2\left(r-\mu-2 \delta_{2}+d\right)-\sigma^{2}} I^{*}\right)^{2} \\
& +\frac{\left(r-\mu-2 \delta_{2}+m\right) \sigma^{2}}{2\left(r-\mu-2 \delta_{2}+m\right)-\sigma^{2}} S^{* 2}+\frac{\left(r-\mu-2 \delta_{2}+d\right) \sigma^{2}}{2\left(r-\mu-2 \delta_{2}+d\right)-\sigma^{2}} I^{* 2}+\frac{\lambda \sigma^{2}}{2} I^{*} \\
:= & F(t) .
\end{aligned}
$$

If

$$
r>\mu+2 \delta_{2}-\delta_{1}+\frac{\sigma^{2}}{2}
$$

then

$$
r-\mu-2 \delta_{2}+m-\frac{\sigma^{2}}{2}>0 \text { and } r-\mu-2 \delta_{2}+d-\frac{\sigma^{2}}{2}>0 .
$$

Therefore,

$$
d V \leq F(t) d t+\frac{\lambda \sigma S}{N}\left(I-I^{*}\right) d B(t) .
$$

Integrating both sides of this from 0 to $t$ yiels

$$
V(t)-V(0) \leq \int_{0}^{t} F(s) d s+\int_{0}^{t} \frac{\lambda \sigma S}{N}\left(I-I^{*}\right) d B(s) .
$$


Set $M_{2}(t):=\int_{0}^{t} \frac{\lambda \sigma S}{V}\left(I-I^{*}\right) d B(s)$, by the large number theorem for martingales, we can obtain

$$
\lim _{t \rightarrow \infty} \frac{M_{2}}{t}=0 \text { a.s. }
$$

which together with (42) implies

$$
\lim _{t \rightarrow \infty} \sup \frac{\int_{0}^{t} F(s) d s}{t} \geq 0 \quad \text { a.s.. }
$$

Choose

$$
\Theta:=\min \left\{r-\mu-2 \delta_{2}+m-\frac{\sigma^{2}}{2}, r-\mu-2 \delta_{2}+d-\frac{\sigma^{2}}{2}\right\}
$$

through dividing both sides of (42) by $t$ and letting $t \rightarrow \infty$, we can obtain

$$
\begin{aligned}
\lim _{t \rightarrow \infty} \sup & \frac{1}{t} \int_{0}^{t}\left(\left(S-\frac{2\left(r-\mu-2 \delta_{2}+m\right)}{2\left(r-\mu-2 \delta_{2}+m\right)-\sigma^{2}} S^{*}\right)^{2}+\left(I-\frac{2\left(r-\mu-2 \delta_{2}+d\right)}{2\left(r-\mu-2 \delta_{2}+d\right)-\sigma^{2}} I^{*}\right)^{2}\right) d \tau \\
& \leq \frac{\Psi}{\Theta}
\end{aligned}
$$

The proof of Theorem (3.2) is completed.

\subsection{Stationary distribution and Ergodic property}

Before giving the main results about the endemic stationary distribution, we first give a definition about stationary distribution [27] and some lemmas.

Definition 3.3. [27] Let $\mathbb{P}\left(t, X_{0}, \cdot\right)$ denote the probability measure induced by $X(t)=$ $(S(t), I(t))$ with initial value $X_{0}=(S(0), I(0))$, that is,

$$
\lim _{t \rightarrow \infty} \mathbb{P}_{X_{0}}(X \in \mathbf{B})=\mathbb{P}\left\{X(t) \in \mathbf{B}: X(0)=X_{0}\right\} \quad \text { for any Borel set } \mathbf{B} \in \mathbb{R}_{+}^{2} .
$$

If there is a probability measure $\pi(\cdot)$ on the measurable apace $\left(\mathbb{R}_{+}^{2}, \mathbf{B}\left(R_{+}^{2}\right)\right)$ such that

$$
\lim _{t \rightarrow \infty} \mathbb{P}_{X_{0}}(X \in \mathbf{B})=\pi(\mathbf{B}) \quad \text { for any } \quad X_{0} \in \mathbb{R}_{+}^{2}
$$

we then say that model (8) has a stationary distribution $\pi(\cdot)$. 
Let $X(t)$ be a regular temporally homogeneous Markov process in $\mathbb{R}_{+}^{2}$ described by the stochastic differential equation

$$
d X(t)=F(X, t) d t+\sum_{r=1}^{2} \sigma_{r}(X) d B_{r}(t)
$$

and the diffusion matrix is defined as follows

$$
A(x)=\left(\left(a_{i j}(x)\right)\right), a_{i j}(x)=\sum_{r=1}^{2} \sigma_{r}^{i}(x) \sigma_{r}^{j}(x) .
$$

For model (8), the diffusion matrix is

$$
A(x)=\frac{\sigma^{2} S^{2} I^{2}}{N^{2}} \operatorname{diag}(1,1) .
$$

Lemma 3.4. [28] Assume that there exists a bounded domain $U \subset \mathbb{R}_{+}^{2}$ with regular boundary, which has the following properties:

(i) In the domain $U$ and some neighborhood thereof, the smallest eigenvalue of the diffusion matrix $A(x)$ is bounded away from zero;

(ii) If $x \in \mathbb{R}_{+}^{2}$, the mean time $\tau$ at which a path issuing from $x$ reaches the set $U$ is finite, and $\sup _{x \in k} E_{x} \tau<\infty$ for every compact subset $K \subset \mathbb{R}_{+}^{2}$.

Lemma 3.5. [28] Suppose that the assumptions in Lemma (3.4) hold, then the Markpv process $X(t)$ has a unique stationary distribution $\pi(\cdot)$. Moreover, if $F(X, t)$ is a function integrable with respect to the measure $\pi$, then

$$
\mathbb{P}_{x}\left\{\lim _{T \rightarrow \infty} \frac{1}{T} \int_{0}^{T} F(X(t)) d t \int_{\mathbb{R}_{+}^{2}} F(t) \pi(d x)\right\}=1
$$

for all $x \in \mathbb{R}_{+}^{2}$.

Applying Theorem 3.2, Lemmas 3.4 and 3.5, we can get:

Theorem 3.6. Consider the stochastic model (8) with initial condition $(S(0), I(0)) \in \mathbb{R}_{+}^{2}$. Suppose that the assumptions in Theorem (3.2) and

$$
0<\Psi<\min \left\{\frac{2\left(r-\mu-2 \delta_{2}+m\right)^{2}}{2\left(r-\mu-2 \delta_{2}+m\right)-\sigma^{2}} S^{*^{2}}, \frac{2\left(r-\mu-2 \delta_{2}+d\right)^{2}}{2\left(r-\mu-2 \delta_{2}+d\right)-\sigma^{2}} I^{*^{2}}\right\},
$$

hold, where $\Psi$ is of the same definitions as in Theorem 3.2. Then there exists a unique stationary distribution $\pi(\cdot)$, and the solution $(S(t), I(t))$ of model (8) is ergodic. 
Proof. To verify (i) of Lemma 3.4, with reference to [29], it is sufficient to show that there exist some neighborhood $\mathrm{U}$ and a nonnegative $C^{2}$-function $V(x)$ such that, for some constants $C>0$

$$
L V(x)<-C \text { for any } x \in \mathbb{R}_{+}^{2} \backslash U
$$

To this end, we use the nonnegative $C^{2}$-function $V(S, I)$ as Theorem 3.2. It follows from Theorem (3.2) that

$$
\begin{aligned}
L V \leq & -\left(r-\mu-2 \delta_{2}+m-\frac{\sigma^{2}}{2}\right)\left(S-\frac{2\left(r-\mu-2 \delta_{2}+m\right.}{2\left(r-\mu-2 \delta_{2}+m\right)-\sigma^{2}} S^{*}\right)^{2} \\
& -\left(r-\mu-2 \delta_{2}+d-\frac{\sigma^{2}}{2}\right)\left(I-\frac{2\left(r-\mu-2 \delta_{2}+d\right)}{2\left(r-\mu-2 \delta_{2}+d\right)-\sigma^{2}} I^{*}\right)^{2}+\Psi .
\end{aligned}
$$

Since

$$
0<\Psi<\min \left\{\frac{2\left(r-\mu-2 \delta_{2}+m\right)^{2}}{2\left(r-\mu-2 \delta_{2}+m\right)-\sigma^{2}} S^{*^{2}}, \frac{2\left(r-\mu-2 \delta_{2}+d\right)^{2}}{2\left(r-\mu-2 \delta_{2}+d\right)-\sigma^{2}} I^{*^{2}}\right\}
$$

the ellipsoid

$$
\begin{aligned}
& \left(r-\mu-2 \delta_{2}+m-\frac{\sigma^{2}}{2}\right)\left(S-\frac{2\left(r-\mu-2 \delta_{2}+m\right)}{2\left(r-\mu-2 \delta_{2}+m\right)-\sigma^{2}} S^{*}\right)^{2} \\
& +\left(r-\mu-2 \delta_{2}+d-\frac{\sigma^{2}}{2}\right)\left(I-\frac{2\left(r-\mu-2 \delta_{2}+d\right)}{2\left(r-\mu-2 \delta_{2}+d\right)-\sigma^{2}} I^{*}\right)^{2}=\Psi
\end{aligned}
$$

lies entirely in $\mathbb{R}_{+}^{2}$. One can then take $U$ as any neighborhood of the ellipsoid such that $\bar{U} \subset \mathbb{R}_{+}^{2}$, where $\bar{U}$ is the closure of $U$. Thus, we have $L V(S, I)<0$ for all $(S, I) \in \mathbb{R}_{+}^{2} \backslash U$, which implies that condition (ii) in Lemma 3.4 is satisfied.

On the other hand, there is $M=\frac{\beta^{2} S^{2} I^{2}}{K^{2}}>0$ such that

$$
\sum_{i, j=1}^{2} a_{i j} \xi_{i} \xi_{j}=\frac{\beta^{2} S^{2} I^{2}}{K^{2}}\left(\left(\xi_{1}\right)^{2}+\left(\xi_{2}\right)^{2}\right) \geq M|\xi|^{2}
$$

for all $(S, I) \in \bar{U}, \zeta \in \mathbb{R}_{+}^{2}$. Thus, by Rayleigh's principle [30], condition (i) in Lemma 3.4 is verified for model (8) has a stationary distribution $\pi(\cdot)$ which is ergodic. 


\section{Numerical results}

In this section, as an example, we give some numerical results to show complex disease dynamic outcomes of the deterministic model (3) (in the case of $\sigma=0$ in model (8)) v.s. its stochastic version (8) with the same set of parameter values by using the Milstein method mentioned in Higham [31]. In this way, model (8) can be rewritten as the following discretization equations:

$$
\left\{\begin{aligned}
S_{k+1}=S_{k} & +\left(r\left(S_{k}+I_{k}\right)\left(1-\frac{S_{k}+I_{k}}{K}\right)-\frac{\beta S_{k} I_{k}}{S_{k}+I_{k}}-(\mu+m) S_{k}\right) \Delta t \\
& -\sigma \frac{S_{k} I_{k}}{S_{k}+I_{k}} \sqrt{\Delta t} \xi_{k}+\frac{\sigma^{2}}{2} \frac{S_{k} I_{k}}{\left(S_{k}+I_{k}\right)}\left(\xi_{k}^{2}-1\right) \Delta t \\
I_{k+1}=I_{k} & +\left(\frac{\beta S_{k} I_{k}}{S_{k}+I_{k}}-(\mu+d) I_{k}\right) \Delta t+\sigma \frac{S_{k} I_{k}}{S_{k}+I_{k}} \sqrt{\Delta t} \xi_{k}+\frac{\sigma^{2}}{2} \frac{S_{k} I_{k}}{\left(S_{k}+I_{k}\right)}\left(\xi_{k}^{2}-1\right) \Delta t,
\end{aligned}\right.
$$

where $\xi_{k}(k=1,2, \ldots, n)$ are independent Gaussian random variables $N(0,1)$.

Considering that $R_{0}^{S}=R_{0}-\frac{\sigma^{2}}{2(\mu+d)}<R_{0}$, if $R_{0}<1$, then $R_{0}^{s}<1$, one can know that if $I(t)$ dies out for the deterministic model (3), then from Theorem 2.2, for the stochastic (8), $I(t)$ almost surely tends to zero exponentially with probability one. In this sense, we only focus on the stochastic disease dynamics in the case of $R_{0}>1$.

The parameter values are chosen as follows. For the Daphnia-microparasite system, the life span in the absence of parasite is $50 \mathrm{~d}$ and hence $d<0.02 / \mathrm{d}$ [1] (here we choose $d=0.01$ [2]). The maximum birth rate in the absence of competition is $r=0.4 / \mathrm{d}[1]$. The disease induced excess mortality rate is $\mu=0 \sim 0.2 / \mathrm{d}$ [1] (here we choose $\mu=0.01$ [2]). And the transmission coefficient is $\beta=1 / \mathrm{d} /$ host [2]. The per-capita emigration rate of the infectious $m \in(0,1) / \mathrm{d}$, here, as an example, we adopt $m=0.1 / \mathrm{d}$.

For the deterministic model (3) and its stochastic model (8), the initial values are taken

as $(S(0), I(0))=(0.9,0.1) \in \Delta$. We can know that the parameters above give in the deterministic case (3), i.e., with $\sigma=0$, a value of the basic reproduction number $R_{0}=$ $9.09091>1, R_{d}=2>1$. Consequently, according to Theorem 1.2, model (3) has a diseasefree equilibrium $E_{0}=(0.25,0)$ which is a saddle point, and an endemic equilibrium point $E^{*}=(0.077028,0.62322)$ which is globally stable. 
Table 1: Parameter values in numerical simulations for model (8)

\begin{tabular}{lll}
\hline Parameters & Value range & Reference \\
\hline$r:$ the maximum per capita birth rate of uninfected hosts & $0.4 / \mathrm{d}$ & {$[1]$} \\
$K$ : The carrying capacity of the habitat & 1.0 & {$[1,2]$} \\
$d:$ The parasite-independent host background mortality & $0 \sim 0.02 / \mathrm{d}$ & {$[1]$} \\
$\beta:$ The transmission coefficient & $1.0 \sim 2.0 / \mathrm{d} / \mathrm{host}$ & {$[2]$} \\
$\mu:$ The parasite-induced excess death rate & $0 \sim 0.2 / \mathrm{d}$ & {$[1]$} \\
$m:$ The per-capita emigration rate of infectious & $0 \sim 1 / \mathrm{d}$ & Estimated \\
\hline
\end{tabular}

Next we focus on the role of noise strength on the resulting dynamics for the SDE model (8). We start our numerical simulation with environmental forcing intensity $\sigma=0.1$ and the numerical simulation results in Fig. 1. In this case, $R_{0}^{s}=9.04545>1$, thus, from Theorem 3.1, we can conclude that the disease will persist in mean. From Fig. 1, we can see that, after some initial transients the solutions $S(t)$ and $I(t)$ of the SDE model (8) fluctuate around the deterministic steady state values $S^{*}=0.077028$ and $I^{*}=0.62322$, respectively (See, Fig. 1(a)). For the sake of learning the effects of the noise on the disease dynamics, we have repeated the simulation 10000 times keeping all parameters fixed and never observing any extinction scenario up to $t=300$. This is confirmed by the histograms in Fig. 1(b), showing the stationary distributions of $S(t)$ and $I(t)$ at $t=300$ for the stochastic model (8). And the numerical method for them can be found in [17]. From Fig. 1(b), one can see that the stationary distributions appear closer to normal distribution.

To see the increased level of non-equilibrium fluctuation in the system dynamics with the increasing noise intensity, we choose $\sigma=0.9$, then $R_{0}^{s}=5.40909>1$. The outcome of the paths and the stationary distributions is presented in Fig.2.

Comparing the numerical results in Fig. 1(b) with 2(b), one can know that, the solution to the SDE model (8) in the persistent case also suggest for higher $\sigma$ (e.g., $\sigma=0.9$ ) that the amplitude of fluctuation is remarkable and the distribution of the solution is skewed 

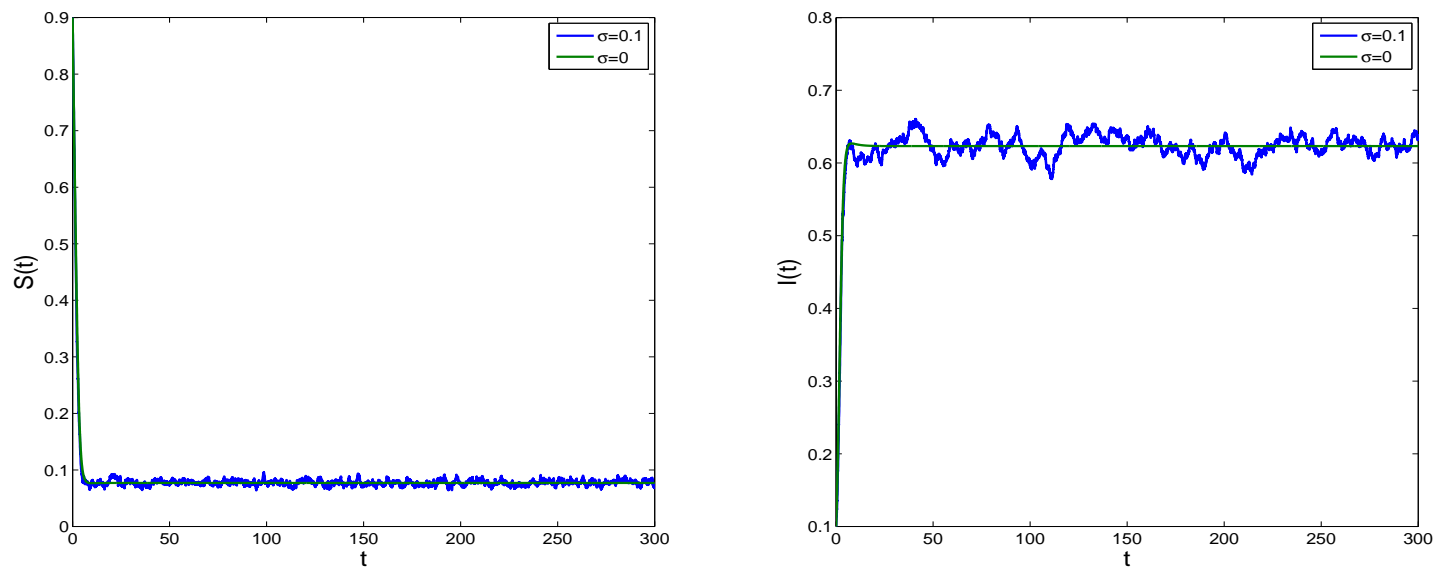

(a) The paths of $S(t)$ and $I(t)$
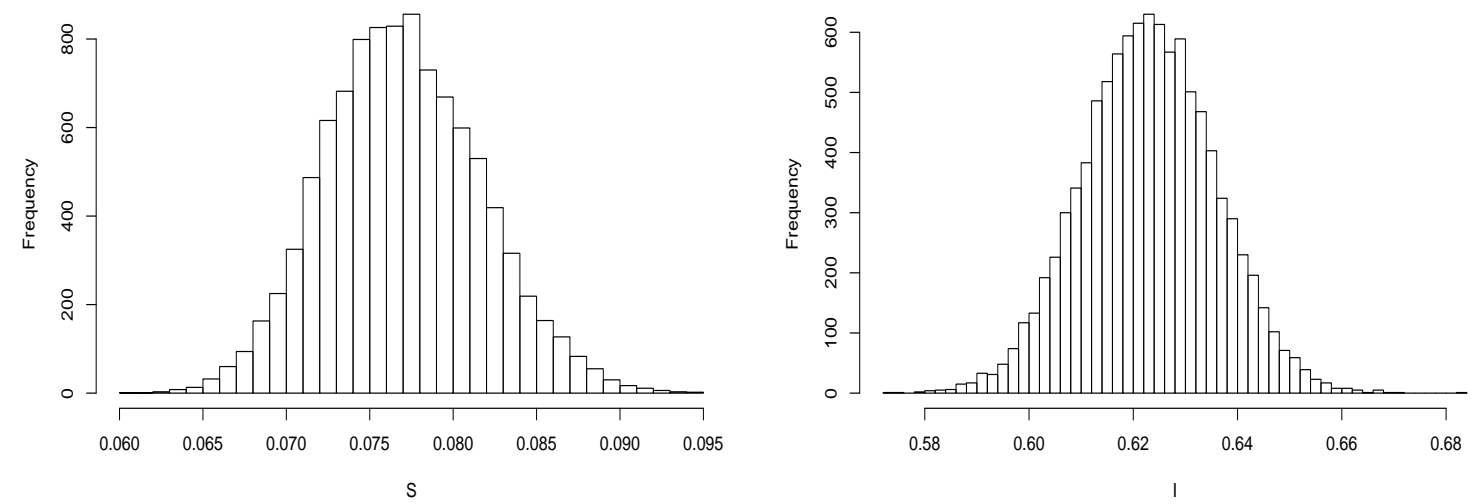

(b) Stationary distributions of $S(t)$ and $I(t)$ at $t=300$

Figure 1: Upper panel: results of one simulation run of the stochastic system (8) with parameter values as mentioned in the text and $\sigma=0.1$ (blue curves). Solution trajectories are plotted along with the solutions of corresponding deterministic model (3) to see the variation of population around the deterministic steady state (green broken curve). Lower panel: stationary distribution of of $S(t)$ and $I(t)$ population obtained at $t=300$ from 10,000 simulation run.

(c.f., Fig. 2(b)) and this fluctuation is also reflected at the stationary distribution as the susceptible $S(t)$ distributing within $(0.009,0.419)$ and the infectious $I(t)$ remaining within the range $(0.094275,0.9988)$. While for lower $\sigma$ (e.g., $\sigma=0.1)$, the amplitude of fluctuation 

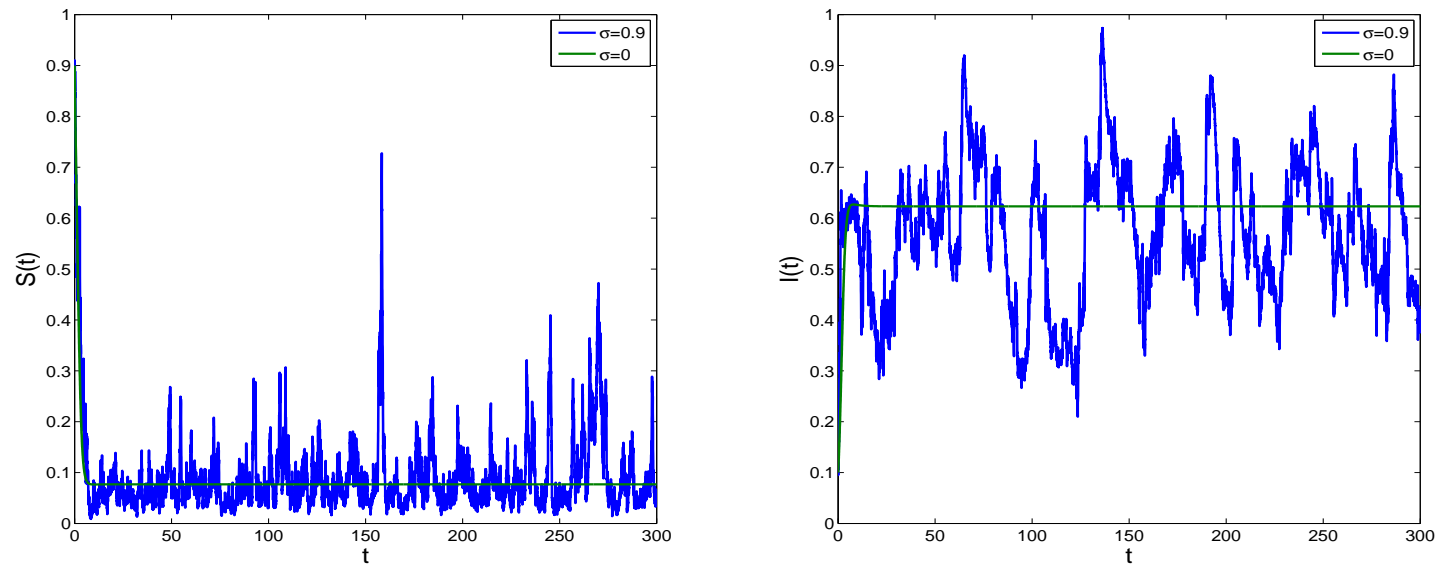

(a) The paths of $S(t)$ and $I(t)$
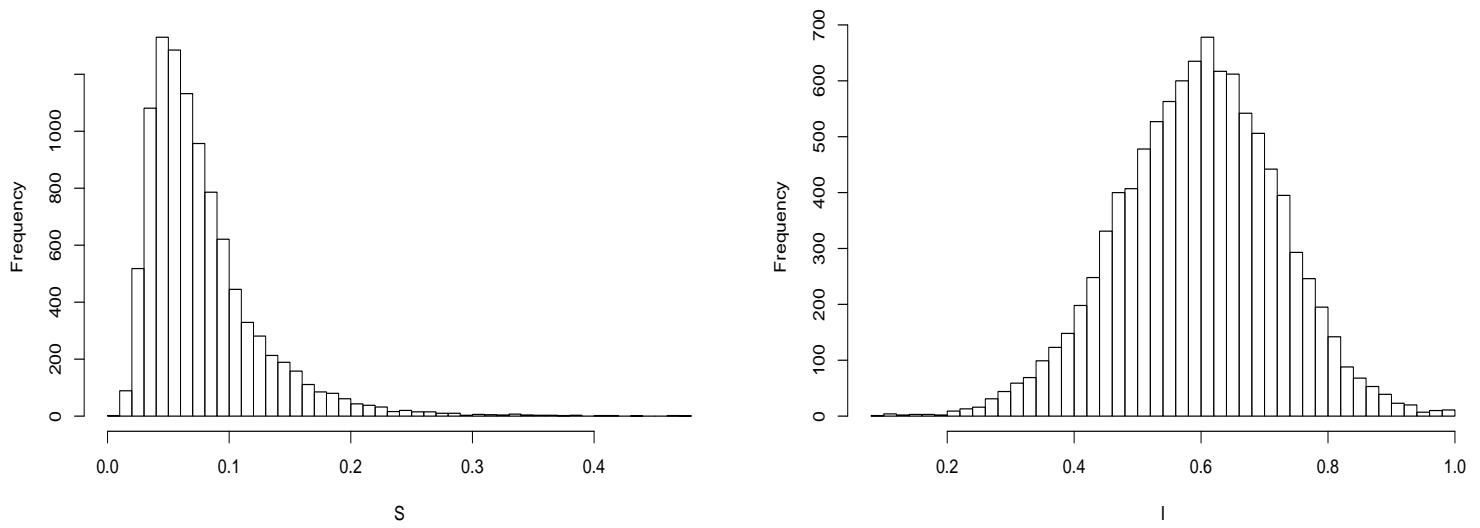

(b) Stationary distributions of $S(t)$ and $I(t)$ at $t=300$

Figure 2: Upper panel: results of one simulation run of the stochastic system (8) with parameter values as mentioned in the text and $\sigma=0.9$ (blue curves). Solution trajectories are plotted along with the solutions of corresponding deterministic model (3) to see the variation of population around the deterministic steady state (green broken curve). Lower panel: stationary distribution of of $S(t)$ and $I(t)$ population obtained at $t=300$ from 10,000 simulation run.

is slightly and the oscillations to be more symmetrically distributed (c.f., Fig. 1(b)), and this fluctuation is also reflected at the stationary distribution as the susceptible $S(t)$ distributing within $(0.068,0.0946)$ and the infectious $I(t)$ remaining within the range $(0.5723,0.6821)$. 
More precisely, when $\sigma=0.1$, the distribution appears closer to a normal distribution (See Fig. 1(b)), but as $\sigma$ increases to 0.9, the distribution is positively skewed (See Fig. 2(b)).

Thanks to the insightful work of Mandal and Banerjee [14], we know that, in the case of noise added models, there is a continuous spectrum of disturbances generated by the added environmental noise terms, and the system is always in tension between deterministic stabilization and random environmental fluctuations. Then we can further study the probabilistic "smoke cloud", which is obtained from the stationary distribution of populations and verify whether the SDE model (8) are stochastically stable systems or not. In Fig. 3, for simplicity, we only show the distributions of $S$ and $I$ obtained through numerical simulation at $t=300$ for $\sigma=0.1$ and $\sigma=0.9$. It is clear that the probability cloud is distributed around the deterministic steady-state $E^{*}=(0.077028,0.62322)$ of model (3) but their dispersion is controlled by the forcing intensity $\sigma$.

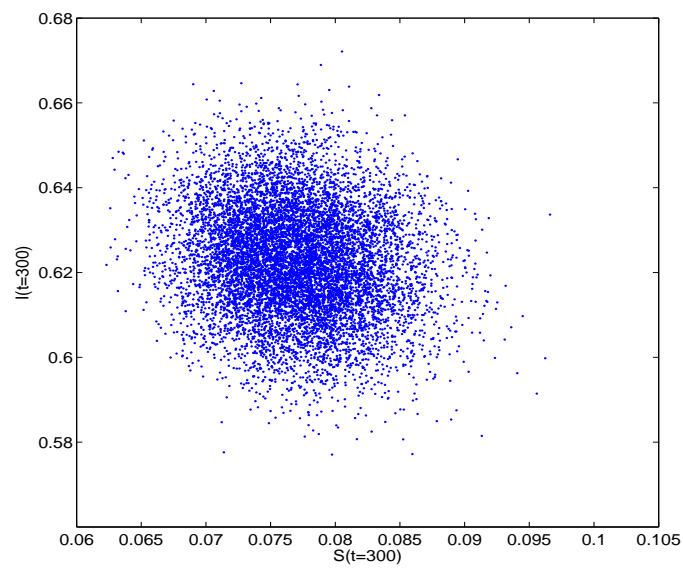

(a) $\sigma=0.1$

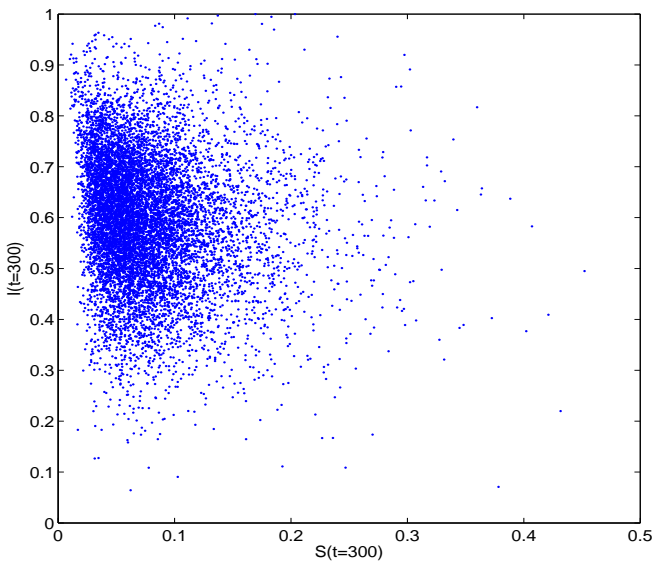

(b) $\sigma=0.9$

Figure 3: Stationary distribution of $S(t)$ and $I(t)$ for the SDE model (8) around deterministic steady-state $E^{*}=(0.077028,0.62322)$ at time $t=300$ for $\sigma=0.1$ (a) and $\sigma=0.9$ (b).

For the sake of learning the effects of the noise on the disease dynamics further, in Figure 4, we show the trends of the evolution of the mean and standard deviation of $I(t)$, respectively. It is observed that in the cases $\sigma_{2}=0.1$ and 0.9 , both the mean (see, Fig.4(a)) and standard deviation (see, Fig.4(b)) ultimately tend to a positive constant; however, in 
the case of $\sigma_{2}=1.6$, both the mean and standard deviation quickly decline and tend to 0 . In addition, from Fig. 4(a), we can also find that when the environmental noises exist, the mean of $I(t)$ is always less than the corresponding deterministic solution for all three cases, and the mean is reduced gradually with the increase of white noise intensity $\sigma$. This means that white noise perturbations of the transmission coefficient $\beta$ can be beneficial to control the spread of disease on average.

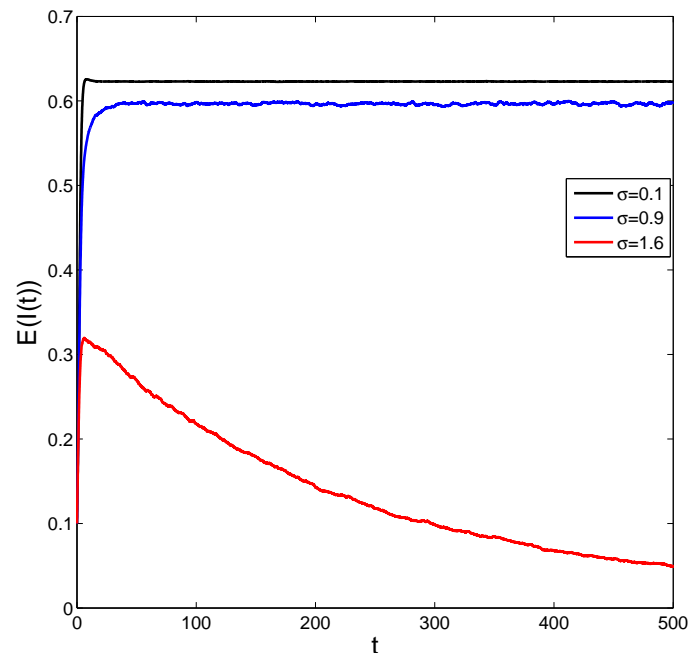

(a)

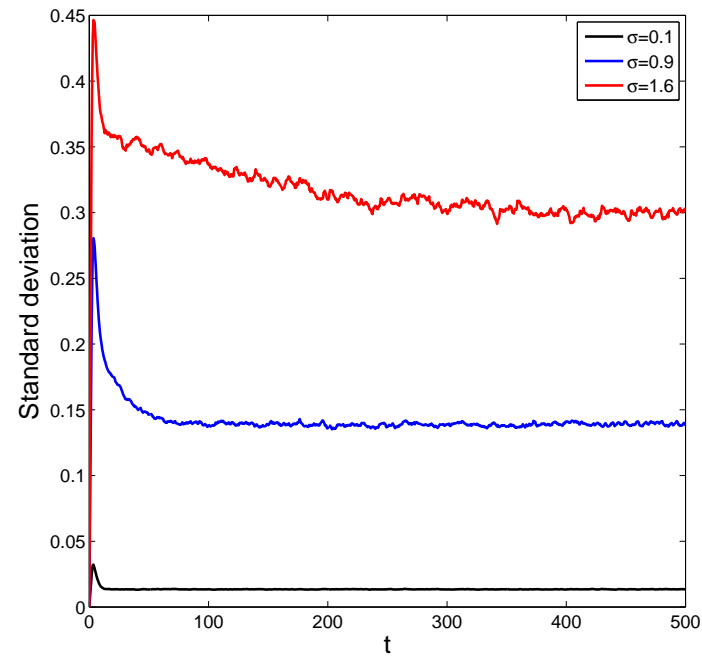

(b)

Figure 4: (a) The mean evolution of infectious $I(t)$ of the stochastic model (8) are graphed for various values of $\sigma$. (b) The corresponding standard deviation evolution of infected individuals of the stochastic model (8).

Finally, we will focus on the stochastic extinction of the disease of model (8). Choose $\sigma=1.6$, then $\sigma^{2}=2.56>\beta=2$, thus from Theorem 2.2, we can conclude that the disease $I(t)$ will die out with probability one. The numerical results are shown in Fig. 5, easy to see that $I(t)$ goes to extinction almost surely, but $S(t)$ survives (c.f., Fig. 5(a)). And from Fig. 5(b), one can know that $S(t)$ of the SDE model (8) tends to $K\left(R_{d}-1\right) / R_{d}=0.5$ and $I(t)$ goes extinct exponentially almost surely. That is, in this case, all solutions of model (8) tend to the absorbing set $(0.5,0)$. This confirms our theoretical findings. 

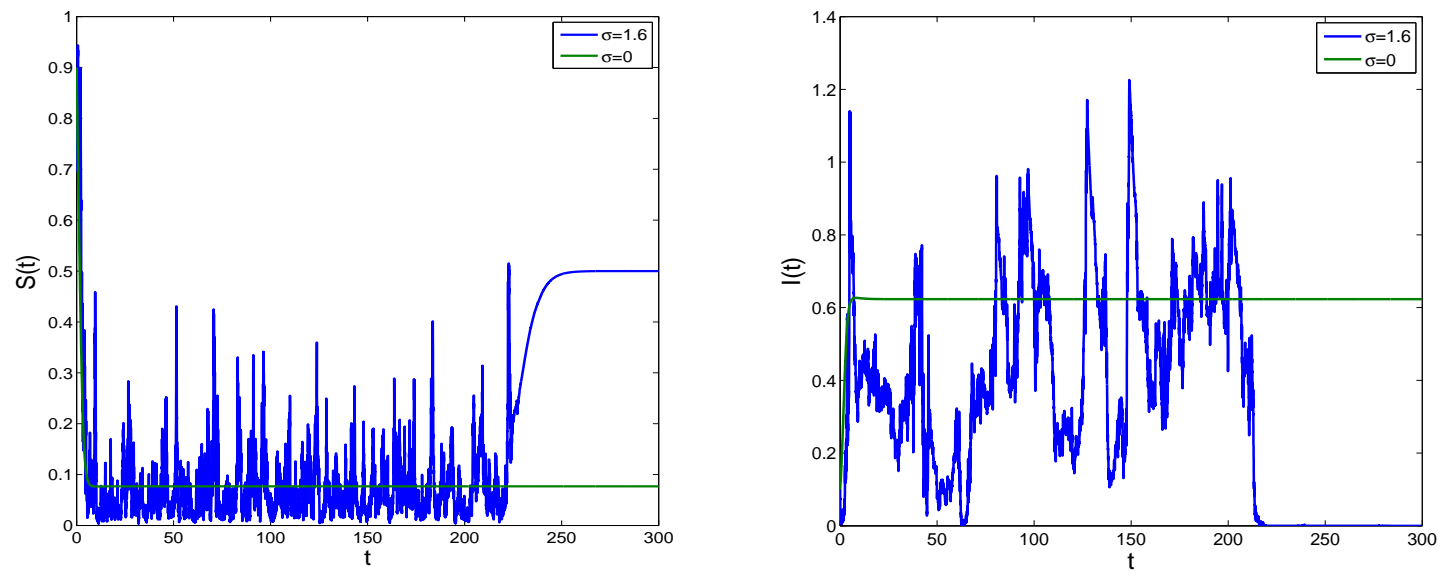

(a) The paths of $S(t)$ and $I(t)$
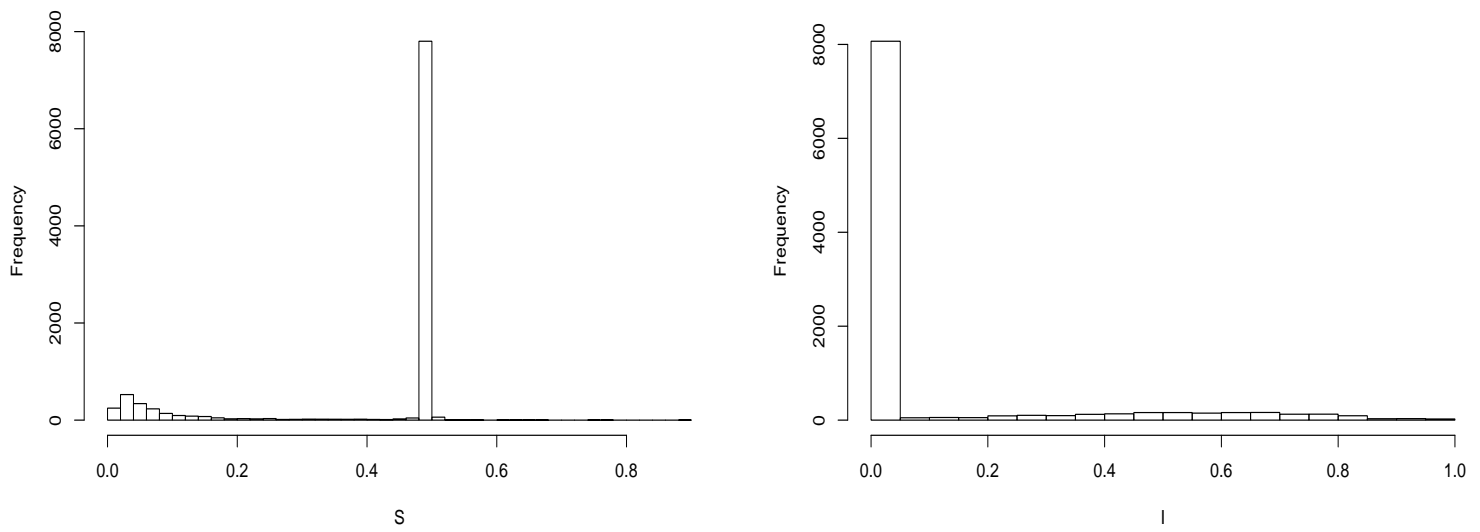

(b) Distributions of $S(t)$ and $I(t)$ at $t=300$

Figure 5: Upper panel: results of one simulation run of the stochastic system (8) with parameter values as mentioned in the text and $\sigma=1.6$ (blue curves). Solution trajectories are plotted along with the solutions of corresponding deterministic model (3) to see the variation of population around the deterministic steady state (green broken curve). Lower panel: distributions of $S(t)$ and $I(t)$ population obtained at $t=300$ from 10,000 simulation run.

\section{Concluding Remarks}

In this paper, we investigate the basic features of a parasite-host epidemiological model in presence of multiplicative noise terms to understand the effects of environmental driving 
forces on the disease dynamics. The value of this study lies in two aspects.

Mathematically, we show that the global dynamics of the stochastic model (8) can be

governed by $R_{0}^{S}=R_{0}-\frac{\sigma^{2}}{2(\mu+d)}$. We have provided the analytic results on the existence of global positive solution, stochastic extinction/permanence, asymptotic stability and ergodic property of the solution of the SDE model (8).

Epidemiologically, we partially provide the effects of the environment fluctuations on the disease spreading to the SDE model (8).

1. Large environment fluctuations can suppress the outbreak of disease: For the deterministic model (3), the disease dies out if $R_{0} \leq 1$ (c.f., Theorem 1.1) and persists if $R_{0}>1$ (c.f., Theorem 1.2). However, this result does not apply to the stochastic model (8) according to Theorem 2.2 since $R_{0}^{S}=R_{0}-\frac{\sigma^{2}}{2(\mu+d)}<R_{0}$, thus it is possible that $R_{0}^{S}<1<R_{0}$. That is, we can find an example when $R_{0}>1$ but $R_{0}^{S}<1$ such that the deterministic model (3) has endemic dynamics while the SDE model (8) has disease-free dynamics with probability one (see Fig. 5). This implies that large environment fluctuations in $I$-class can suppress the outbreak of disease.

2. The distributions governed by $R_{0}^{S}$ : If the noise intensity $\sigma$ is small enough to imply that $R_{0}^{S}>1$, from Theorem 3.1 and 3.2, one can know that the noise can force the solutions of model (8) to fluctuate around the deterministic steady state values $E^{*}=$ $\left(S^{*}, I^{*}\right)$ of model (3), respectively, and the irregularity of stochastic variation and range of fluctuation increase with the increase in the magnitude of $\sigma$ (c.f., Fig. 1, 2 and 3). In addition, as suggested by Theorems 3.6, the SDE model (8) has an endemic stationary distribution (See, Fig. 1(b) and 2(b)) which leads to the stochastically persistence of the disease. But if the noise intensity $\sigma$ is large enough to imply that $R_{0}^{S}<1$, Theorem 2.2 shows that the disease will die out with probability one (See, Fig. 5(a)), and $S(t)$ tends to $K\left(R_{d}-1\right) / R_{d}$ and $I(t)$ goes extinct exponentially almost surely (See, Fig. $5(\mathrm{~b})$ ). That is, in this case, all solutions of model (8) tend to an absorbing set $(0.5,0)$.

3. The noise can be beneficial to control the disease spread on average: From the numerical results of the trends of the evolution of the mean and standard deviation of 
$I(t)$ in Fig. 4, we can know that the average level of the number of infected individuals $I(t)$ always decreases with the increase of noise intensity. This means that white noise perturbations can be beneficial to control the spread of disease on average.

\section{Acknowledgements}

This research was supported by the National Science Foundation of China (61373005).

\section{References}

[1] D. Ebert, M. Lipsitch, and K.L. Mangin. The effect of parasites on host population density and extinction: experimental epidemiology with daphnia and six microparasites. The American Naturalist, 156(5):459-477, 2000.

[2] T. Hwang and Y. Kuang. Deterministic extinction effect of parasites on host populations. Journal of Mathematical Biology, 46(1):17-30, 2003.

[3] T. Hwang and Y. Kuang. Host extinction dynamics in a simple parasite-host interaction model. Math. Biosc. Eng, 2(743):51, 2005.

[4] F. Berezovsky, G. Karev, B. Song, and C. Castillo-Chavez. A simple epidemic model with surprising dynamics. Mathematical Biosciences and Engineering, 2(1):133-152, 2005.

[5] Y. Kang and C. Castillo-Chavez. A simple epidemiological model for populations in the wild with allee effects and disease-modified fitness. Discrete and Continuous Dynamical Systems. Series B, 19(1):89, 2014.

[6] Y. Cai and W. Wang. Dynamics of a parasite-host epidemiological model in spatial heterogeneous environment. Discrete and Continuous Dynamical Systems. Series B, 20(4):989-1013, 2015. 
[7] X. Mao, G. Marion, and E. Renshaw. Environmental brownian noise suppresses explosions in population dynamics. Stochastic Processes and Their Applications, 97(1):95$110,2002$.

[8] M. Liu and K. Wang. Survival analysis of stochastic single-species population models in polluted environments. Ecological Modelling, 220(9):1347-1357, 2009.

[9] T. Britton. Stochastic epidemic models: a survey. Mathematical Biosciences, 225(1):24$35,2010$.

[10] A. Gray, D. Greenhalgh, L. Hu, X. Mao, and J. Pan. A stochastic differential equation sis epidemic model. SIAM Journal on Applied Mathematics, 71(3):876-902, 2011.

[11] A. Lahrouz, L. Omari, and D. Kiouach. Global analysis of a deterministic and stochastic nonlinear sirs epidemic model. Nonlinear Analysis: Modelling and Control, 16(1):59-76, 2011.

[12] M. Liu, K. Wang, and Q. Wu. Survival analysis of stochastic competitive models in a polluted environment and stochastic competitive exclusion principle. Bulletin of Mathematical Biology, 73(9):1969-2012, 2011.

[13] M. Liu and K. Wang. Persistence, extinction and global asymptotical stability of a nonautonomous predator-prey model with random perturbation. Applied Mathematical Modelling, 36(11):5344-5353, 2012.

[14] P.S. Mandal and M. Banerjee. Stochastic persistence and stationary distribution in a holling-tanner type prey-predator model. Physica A: Statistical Mechanics and its Applications, 391(4):1216-1233, 2012.

[15] Y. Zhao, D. Jiang, X. Mao, and A. Gray. The threshold of a stochastic sirs epidemic model in a population with varying size. Discrete and Continuous Dynamical Systems. Series B, 20(4):1277-1295, 2015. 
[16] Y. Cai, Y. Kang, M. Banerjee, and W. Wang. A stochastic epidemic model incorporating media coverage. Communications in Mathematical Sciences, 14:892-910, 2016.

[17] Y. Cai, Y. Kang, M. Banerjee, and W. Wang. A stochastic sirs epidemic model with infectious force under intervention strategies. Journal of Differential Equation$s, 259(12): 7463-7502,2015$.

[18] D. Li, J. Cui, M. Liu, and S. Liu. The evolutionary dynamics of stochastic epidemic model with nonlinear incidence rate. Bulletin of Mathematical Biology, 77(9):1705-1743, 2015.

[19] Y. Zhao, S. Yuan, and J. Ma. Survival and stationary distribution analysis of a stochastic competitive model of three species in a polluted environment. Bulletin of Mathematical Biology, 77:1285-1326, 2015.

[20] Y. Xu, X. Jin, and H. Zhang. Parallel logic gates in synthetic gene networks induced by non-gaussian noise. Physical Review E, 88:052721, Nov 2013.

[21] Y. Xu, J. Feng, J. Li, and H. Zhang. Stochastic bifurcation for a tumor-immune system with symmetric lévy noise. Physica A: Statistical Mechanics and its Applications, 392(20):4739-4748, 2013.

[22] Y. Xu, J. Feng, J. Li, and H. Zhang. Lévy noise induced switch in the gene transcriptional regulatory system. Chaos: An Interdisciplinary Journal of Nonlinear Science, 23(1):013110, 2013.

[23] Y. Xu, Y. Li, and D. Liu. Response of fractional oscillators with viscoelastic term under random excitation. Journal of Computational and Nonlinear Dynamics, 9(3):031015, 2014.

[24] Y. Xu, Y. Zhu, J. Shen, and J. Su. Switch dynamics for stochastic model of genetic toggle switch. Physica A: Statistical Mechanics and its Applications, 416:461-466, 2014. 
[25] Z. Wang, Y. Xu, and H. Yang. Lévy noise induced stochastic resonance in an fhn model. Science China Technological Sciences, 59:371-375, 2016.

[26] C. Ji and D. Jiang. Threshold behaviour of a stochastic sir model. Applied Mathematical Modelling, 38(21):5067-5079, 2014.

[27] J.M. Harrison and S.I. Resnick. The stationary distribution and first exit probabilities of a storage process with general release rule. Mathematics of Operations Research, 1(4):347-358, 1976.

[28] R. Khasminskii. Stochastic stability of differential equations, volume 66. Springer Science \& Business Media, 2011.

[29] C. Zhu and G. Yin. Asymptotic properties of hybrid diffusion systems. SIAM Journal on Control and Optimization, 46(4):1155-1179, 2007.

[30] G. Strang. Linear algebra and its applications. Technical report, 2006.

[31] D.J. Higham. An algorithmic introduction to numerical simulation of stochastic differential equations. SIAM Review, 43(3):525-546, 2001. 